\title{
EUROPEAN ADAPTATION TO EXPEDITIONARY WARFARE: IMPLICATIONS FOR THE U.S. ARMY
}

Andrew Doyman

November 2002 